\title{
Impacto de COVID-19 en la enfermedad cardiovascular
}

\author{
Impact of COVID-19 on cardiovascular disease
}

Cristhian Felipe Ramírez-Ramos ${ }^{1 *}$ ¿cvlac , Diego Mauricio Vanegas ${ }^{2}$, Diego A. Ramírez-Méndez ${ }^{3}$,

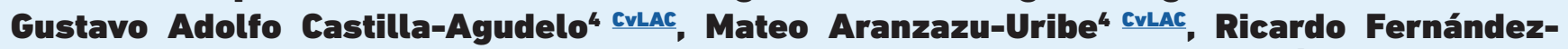
Ruiz ${ }^{5}$, Carlos Esteban Uribe-Londoño6, Luz Adriana Ocampo-Aristizabal7, Álvaro Mauricio Quintero-Ossa ${ }^{8 \text { CvLAC }}$, Catalina Gallego9 CvLAC, Clara Inés Saldarriaga-Giraldo ${ }^{10}$

Fecha correspondencia:

Recibido: mayo 12 de 2020.

Revisado: septiembre 16 de 2020.

Aceptado: septiembre 28 de 2020.

Forma de citar:

Ramírez-Ramos CF, Vanegas DM, Ramírez-Méndez DA, CastillaAgudelo GA, Aranzazu-Uribe M, Fernandez-Rui R et al. Impacto de COVID-19 en la enfermedad cardiovascular. Rev CES Med. 2020. Especial COVID-19: 128-136.

$\underline{\text { Open access }}$

(C) Derecho de autor

Licencia creative commons

Ética de publicaciones

Revisión por pares

Gestión por Open Journal System DOl: http://dx.doi.org/10.21615/ cesmedicina.34.COVID-19.18

ISSN 0120-8705

e-ISSN 2215-9177

Comparte

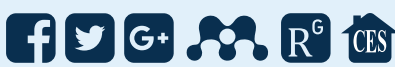

\section{Resumen}

La enfermedad por coronavirus-2019 (COVID-19) es una enfermedad infecciosa cuya sintomatología inicial, en la mayoría de las ocasiones, se manifiesta con compromiso del tracto respiratorio. El virus que causa dicha enfermedad se denomina síndrome respiratorio agudo severo por coronavirus 2 (SARS-CoV2). Si bien la manifestación grave más frecuente de la infección parece ser la neumonía, se han documentado diversas implicaciones cardiovasculares. Las personas que cursan con dicha infección y que tienen una enfermedad cardiovascular preexistente tienen un riesgo mayor de cuadros graves y mortalidad. Existen asociaciones directas o indirectas de la infección con injuria miocárdica, arritmias, enfermedad tromboembólica venosa y miocarditis. Los tratamientos en investigación pueden tener efectos adversos en el sistema eléctrico del corazón; además, algunos medicamentos de uso crónico (como los inhibidores del sistema renina angiotensina aldosterona) se les ha otorgado un polémico papel en la virulencia del microorganismo. La alta demanda en atención en salud que requieren los pacientes infectados por SARS-CoV 2 puede comprometer la asistencia de pacientes cardiovasculares sin dicha infección y dejar en riesgo de exposición al personal sanitario. Se presenta una revisión sobre los aspectos más llamativos donde estas dos condiciones interaccionan.

Palabras claves: Coronavirus; Terapia cardiovascular; Complicaciones.

\begin{abstract}
Coronavirus-2019 disease (COVID-19) is an infectious disease which initial symptoms, in most cases, manifest with respiratory tract compromise. The virus that causes said disease is called severe acute respiratory syndrome by coronavirus 2 (SARS-CoV 2), although the most frequent serious manifestation of the infection appears to be pneumonia, various implications have been documented from the cardiovascular point of view. People who have this infection and a pre-existing cardiovascular disease have an increased risk of severe symptoms and mortality. There are direct or indirect associations of the infection with myocardial injury, arrhythmias, venous thromboembolic disease and myocarditis. Investigational treatments may have adverse effects on the heart's electrical system. In addition, some medications of chronic use (such as renin angiotensin aldosterone system
\end{abstract}




\section{Sobre los autores:}

1. Internista, fellow de cardiología Universidad Pontificia Bolivariana, Clínica CardioVID. Medellín Colombia.

2. Internista y cardiólogo. Fellow de cardiología intervencionista Universidad Pontificia Bolivariana, Clínica CardioVID.

3. Médico Universidad Surcolombiana, Medellín Colombia.

4. Residente de Medicina Interna, Universidad Pontificia Bolivariana. Medellín Colombia.

5. Internista, cardiólogo y ecocardiografísta, departamento de cardiología no invasiva Universidad Pontificia Bolivariana y Clínica CardioVID. Medellín Colombia.

6. Internista, cardiólogo, hemodinamista, jefe del laboratorio de hemodinámica Universidad Pontificia Bolivariana y Clínica CardioVID. Medellín Colombia.

7. Internista, cardióloga, ecocardiografísta, Universidad Pontificia Bolivariana, grupo de enfermedad cardiaca congénita del adulto Clínica CardioVID. Medellín Colombia. inhibitors), have been given a controversial role in the virulence of the organism. The high demand in health care required by patients infected with SARS-CoV 2 can compromise the care of cardiovascular patients without said infection, in addition to leaving healthcare personal at risk of exposure. Therefore, a review is presented on the most striking aspects where these two conditions interact.

Keywords: Coronavirus; Cardiovascular therapy; Complications.

\section{Introducción}

El 31 de diciembre del 2019, autoridades locales en China reportaron un grupo de pacientes con neumonía de etiología desconocida que desde el punto de vista epidemiológico se vinculó a un mercado de animales silvestres y comida de mar en la cuidad de Wuhan, en la provincia de Hubei en China. Semanas después se confirmó que un microorganismo viral perteneciente a la familia Coronaviridae, genero betacoronavirus denominado síndrome respiratorio agudo severo por coronavirus 2 (SARS-CoV 2) era la etiología de este cuadro conocido como enfermedad por coronavirus 2019 (COVID-19, acrónimo del inglés coronavirus disease 2019). A partir de ahí se presentó una rápida propagación de la infección en China, y posteriormente a nivel mundial, llevando a que la Organización Mundial de la Salud declarara dicho brote como una pandemia, por lo que fue necesario tomar medidas drásticas como confinamiento social y cuarentena de países enteros en un hecho sin precedentes. Una proporción importante de pacientes que presentaron síntomas compatibles con COVID-19 reportan comorbilidades cardiovasculares en su historial médico $(1,2)$, lo cual se relaciona con mayor gravedad y mortalidad (3). Con lo anterior se hace importante abordar los aspectos relacionados a pacientes con enfermedad cardiovascular desde el punto de vista clínico y terapéutico. Presentamos una revisión narrativa de la experiencia publicada a la fecha.

\section{Prevalencia de comorbilidades}

En una serie de 44672 casos de pacientes con la infección en China, donde se incluyeron los casos leves, 4,2 \% tenían el antecedente de enfermedad cardiovascular y $12,8 \%$ tenía hipertensión arterial. En esta población, el 13,8 \% presentó cuadros graves sin mortalidad y $4,7 \%$ tuvo un curso crítico con una mortalidad de $49 \%$. En los pacientes que presentaron muerte como desenlace el $22,7 \%$ tenía historia de enfermedad cardiovascular (4).

Chen et al. encuentran que el $40 \%$ tenía enfermedad cardiovascular o cerebrovascular (5). Guan et al. (1) reportan una prevalencia de $15 \%$ para hipertensión arterial, $7,4 \%$ de diabetes, $2,3 \%$ de enfermedad coronaria y $1,4 \%$ de enfermedad cardiovascular. Wang et al. (3) publican las características de pacientes hospitalizados por neumonía en Wuhan; en los pacientes trasferidos a la unidad de cuidado intensivo por complicaciones la presencia de comorbilidades fue de 46,4 \% (cuadro 1).

Un metaanálisis de los estudios anteriores muestra una prevalencia de 21,1\% para hipertensión arterial (IC 95 \% 13-27,2 \%), 9.7 \% para diabetes (IC 95 \% 7,2-12,2 \%) y $8,4 \%$ de enfermedad cardiovascular (IC $95 \%$ 3,8-13,8 \%) (10). Entre los pacientes con cuadros graves se encontró un aumento del riesgo de 2,36 veces asociado a la presencia de hipertensión (IC $95 \%$ 1,46-3,83) y de 3,46 veces para enfermedad cardiovascular (IC $95 \%$ 1,88-6,22). 
8. Internista y cardiólogo, Universidad Pontificia Bolivariana, magister en epidemiología. Jefe del servicio de trasplante cardiaco Clínica CardioVID.

9. Internista y cardióloga, Universidad Pontificia Bolivariana, jefe de la unidad de cuidados coronarios Clínica CardioVID. Medellín Colombia.

10. Internista, cardióloga y especialista en insuficiencia cardiaca jefe del departamento de cardiología Universidad Pontificia Bolivariana y Clínica CardioVID. Docente Universidad de Antioquia.

Han sido encontrados niveles elevados de troponina en pacientes con COVID-19, con importantes diferencias entre los pacientes que mueren.
Cuadro 1. Enfermedades crónicas reportadas en diferentes estudios

\begin{tabular}{lcccccc}
\hline \multicolumn{1}{c}{ Estudio } & $\begin{array}{c}\text { Pacientes } \\
(\boldsymbol{n})\end{array}$ & $\begin{array}{c}\text { Edad } \\
\text { (años) }\end{array}$ & $\begin{array}{c}\text { Hipertensión } \\
(\%)\end{array}$ & $\begin{array}{c}\text { Diabetes } \\
\text { (\%) }\end{array}$ & $\begin{array}{c}\text { Enfermedad } \\
\text { cardiovascular (\%) }\end{array}$ & $\begin{array}{c}\text { Mortalidad } \\
\text { (\%) }\end{array}$ \\
\hline Huang (2) & 41 & 49 & 15 & 20 & 15 & 15 \\
\hline Kui (6) & 137 & 57 & 9,5 & 10,2 & 7,3 & 11,7 \\
\hline Liu (7) & 12 & 53 & 25 & 16,7 & 33 & - \\
\hline Wang (3) & 138 & 56 & 31 & 10,1 & 14,5 & 4,3 \\
\hline Zhang (8) & 140 & 57 & 30 & 12,1 & 5 & - \\
\hline Guan (1) & 1099 & 47 & 14,9 & 7,4 & 2,5 & 1,4 \\
\hline CDC China (9) & 44672 & 46 & 12,8 & 5,3 & 4,1 & 2,3 \\
\hline
\end{tabular}

\section{Factores de riesgo asociados a mortalidad}

Los factores de riesgo de mortalidad descritos han sido: la edad mayor de 65 años, las comorbilidades, la presencia de síndrome de dificultad respiratorio agudo (11), el puntaje de SOFA (siglas en inglés de Sequential Organ Failure Assessment), los niveles altos de dímero $D$ al ingreso (12), la presencia de enfermedad cardiovascular y los mayores niveles de troponina, mioglobina, proteína $\mathrm{C}$ reactiva, ferritina e interleuquina 6 (13).

\section{Secuelas cardiovasculares asociadas con COVID-19}

\section{Injuria miocárdica, miocarditis y síndromes coronarios agudos}

Han sido encontrados niveles elevados de troponina en pacientes con COVID-19, con importantes diferencias entre los pacientes que mueren (13). En un metaanálisis de cuatro estudios y 341 pacientes, la diferencia entre los niveles de troponina I fue significativamente mayor en los que presentaron cuadros graves (25,6 ng/L, IC $95 \%$ 6,8-44,5) (14). Los estudios publicados estiman una presencia de injuria miocárdica de $7-28 \%$ en los pacientes hospitalizados $(1,3,12,15)$, algo significativamente más frecuente en los pacientes que fallecen ( $59 \%$ vs. $1 \%, p<0,0001$ ) o que requiere manejo en unidad de cuidados intensivos (UCI) $(22,2 \%$ vs. 2,0 \%, p<0,001) (12).

Los pacientes con injuria miocárdica tienden a ser mayores, con más comorbilidades de base (hipertensión, diabetes, enfermedad coronaria y falla cardiaca). En todos los estudios la presencia de injuria miocárdica se relacionó con peores resultados incluyendo muerte y admisión a UCI $(3,15)$. El tiempo medio de desarrollo de la injuria miocárdica es 15 días después del inicio de los síntomas y después de la presencia de síndrome de dificultad respiratorio agudo (12). Entre las 68 muertes de una serie de 150 individuos, el $7 \%$ de estas se atribuyeron a la presencia de miocarditis con falla circulatoria y se consideró que en el 33 \% la inflamación miocárdica tuvo un rol en el desenlace adverso (13). En un pequeño estudio de pacientes recuperados con síntomas cardiacos, la imagen de resonancia magnética reveló compromiso miocárdico en el 58 \% de los casos, dado por edema miocárdico o cicatrices (16). Puntmann et al. reportan los resultados de un estudio de cohortes de pacientes recuperados recientemente de la infección. Aquí, 100 pacientes se estudiaron con resonancia magnética cardiaca; la edad promedio fue de 49 años, 53 \% eran hombres, 33 \% recibió manejo hospitalario y 71 \% tuvo niveles detectables de troponina de alta sensibilidad. En el $78 \%$ de pacientes se encontraron anormalidades en los estudios de resonancia, con $60 \%$ de la población demostrando cambios de inflamación activa. Los hallazgos fueron independientes de las condiciones preexistentes, 
Si bien se esperaba un aumento en la tasa de presentación de los síndromes coronarios agudos, lo que se ha reportado de manera llamativa ha sido una disminución tanto de las hospitalizaciones como de los procedimientos de intervención coronaria percutánea. la severidad y el curso global de la enfermedad (17). Lo anterior, soporta la necesidad de seguimiento a largo plazo en el cuidado cardiovascular de los pacientes con infección por SARS-CoV2, teniendo la incertidumbre del impacto en la función cardiaca a largo plazo.

Con respecto a los síndromes coronarios agudos hay un comportamiento particular. Si bien se esperaba un aumento en la tasa de presentación de este grupo de enfermedades, lo que se ha reportado de manera llamativa ha sido una disminución tanto de las hospitalizaciones como de los procedimientos de intervención coronaria percutánea. En Inglaterra se reporta una reducción del 43 \% de los estudios invasivos por infarto agudo del miocardio con elevación del ST (IAMCEST) y un incremento el tiempo desde el inicio de síntomas a la llegada del hospital como del tiempo puerta-balón sin cambios en la mortalidad (18). En Estados Unidos se han reportado reducciones del número de activaciones de la red de IAMCEST del $29 \%$, de $20 \%$ para los procedimientos de angioplastia primaria (19) y de las hospitalizaciones por esta condición entre 38-48 \% (20). La disminución reportada en Italia y España ha sido de aproximadamente $40 \%$ (21). Un aspecto que genera gran preocupación es que se han incrementado los reportes de las complicaciones relacionadas al infarto, las cuales son muy infrecuentes en la época actual del manejo de la revascularización (22).

Los registros de intervención coronaria percutánea primaria durante la pandemia en pacientes con la infección han mostrado algunos aspectos importantes de resaltar. En Inglaterra los datos de un centro, en un modelo de comparación que incluye 39 pacientes positivos y 76 pacientes negativos para SASR-CoV2 se encontró una tasa de ventilación mecánica de $12 \%$ vs. 6,6 \% y de mortalidad hospitalaria 17,9\% vs. $6,5 \%$ para el primer y segundo grupo, respectivamente. Como hallazgo importante se encontró alta carga trombótica en $75 \%$ de los pacientes con la infección vs. $31 \%$ en los no infectados (23). Una serie de casos de seis hospitales en Nueva York identifica 18 pacientes con COVID-19 y elevación del segmento ST. La edad promedio fue 63 años, 83 \% hombres, solo un tercio de la población presentó dolor torácico y el 39 \% se presentó con choque. De los nueve pacientes llevados a angiografía coronaria, 67 \% tenía enfermedad coronaria obstructiva y en cinco realizó intervención percutánea. El 72 \% murió durante la hospitalización (24).

\section{Arritmias cardiacas}

Las palpitaciones fueron parte de los síntomas de presentación en 7,3 \% en el estudio de Liu et al. (6). En una cohorte de pacientes hospitalizados, las arritmias estuvieron presentes en el $16 \%$, siendo más frecuentes en los ingresados a la $\mathrm{UCI}(44 \%$ vs. 6,9\%) (3). Guo et al. encuentran una frecuencia de 5,9\% de taquicardia ventricular sostenida y fibrilación ventricular (25).

\section{Falla cardiaca}

En la cohorte de Zhou et al., la falla cardiaca se observó en el $23 \%$ de los pacientes, siendo más frecuente en los que no sobrevivieron (51 \% vs. 11,7 \%) (12). En una serie más pequeña de 21 pacientes críticamente enfermos en el estado de Washington la incidencia de disfunción sistólica y choque cardiogénico fue de 33 \% (26). 
Si bien la ribavirina no tiene toxicidad cardiovascular conocida, el lopinavir/ritonavir prolonga el PR y el QT. Estos medicamentos además afectan las dosis de los anticoagulantes.

\section{Enfermedad tromboembólica venosa}

Aunque no hay series publicadas, los reportes ponen de manifiesto anormalidades de los parámetros de la coagulación en pacientes con cuadros graves. El dímero $D$ > $1 \mathrm{~g} / \mathrm{L}$ se encontró relacionado de manera importante con mortalidad intrahospitalaria (OR 18,4 IC 95 \% 2,6-128) (12).

\section{Aspectos terapéuticos y sus interacciones}

\section{Inhibidores del sistema renina angiotensina aldosterona}

El receptor de la enzima convertidora de angiotensina 2 (ACE2) sirve como un receptor viral. Se ha documentado una expresión extensa a nivel cardiaco, renal y epitelio alveolar pulmonar (células blanco del SARS-CoV 2). Pese a la notoria homología estructural entre la ACE y ACE2, su sitio de acción enzimático difiere y los IECA de uso clínico no afectan de manera directa esta molécula. Los pocos estudios clínicos muestran que el uso de IECA no afecta los niveles de angiotensina, la ACE2 plasmática ni urinaria (27). A pesar de toda esta incertidumbre teórica hay un claro potencial de daño con la suspensión de IECA o ARA-II por un aumento del riesgo de descompensación. Los estudios muestran una evidencia neutra sin afectar el desenlace con el uso de estos medicamentos en pacientes con infección por SARS-CoV 2 (27)

\section{Cloroquina y azitromicina}

La cloroquina bloquea la infección viral aumentado el pH endosomal requerido para la fusión y ha demostrado actividad inhibitoria in vitro (28). Al igual que la hidroxicloroquina tiene un potencial de toxicidad miocárdica intermedio - tardío (29). La toxicidad se presenta como una cardiomiopatía dilatada o restrictiva o anormalidades del sistema de la conducción que se piensa son causadas por la inhibición intracelular de enzimas lisosomales. Por inhibición en la CYP2D6, los betabloqueadores que tienen metabolismo por esta enzima tienen niveles aumentados por lo que deben monitorizarse de manera estrecha la presión arterial y la frecuencia cardiaca. La cloroquina y la hidroxicloroquina generan prolongación del QT con un riesgo condicional para torsade des pointes en pacientes con anormalidades electrolíticas o con uso concomitante de otras moléculas que prolonguen el QT (como azitromicina). No se cuentan con reportes de muerte arrítmica con el uso de cloroquina, pero posiblemente sea por la falta de un sistema de vigilancia activa en muchos países. La azitromicina tiene evidencia de inhibición de canales iKr de potasio con una tasa de muerte por arritmias estimada de 47 muertes por 1 millón de prescripciones (30). En un estudio publicado el régimen de cloroquina o hidroxicloroquina con o sin macrólido mostró una falta de beneficio en esta población con una tendencia a incremento en la mortalidad intrahospitalaria; sin embargo, de manera posterior y al tratar se realizar un análisis detallado de los hallazgos, la revista y los autores presentaron una retracción por la imposibilidad de suministrar la información completa de los pacientes incluidos, lo que dejo en duda la veracidad de los hallazgos (30).

\section{Terapia antiviral}

La ribavirina y el remdesivir son dos agentes que se unen al sitio activo de la RNA polimerasa viral, mientras que el lopinavir/ritonavir inhibe la replicación RNA viral con un efecto sinérgico in vitro cuando se combina con ribavirina (31). Si bien la ribavirina no tiene toxicidad cardiovascular conocida, el lopinavir/ritonavir prolonga el PR y el QT. Estos medicamentos además afectan las dosis de los anticoagulantes: la ribavirina afecta la dosificación de la warfarina y el lopinavir/ritonavir puede requerir que se reduzcan o eviten el rivaroxaban, el apixaban u otros medicamentos metabolizados por el CYP3A4. También el lopinavir/ritonavir puede influenciar la 
Debe considerarse tamización a todos los donantes, dada la prevalencia de infección asintomática por SARS-CoV 2. actividad de los inhibidores de la P2Y12 por inhibición de la CYP3A4 generando disminución de las concentraciones de clopidogrel y prasugrel mientras aumenta las de ticagrelol. Si se requiere antiagregación plaquetaria debe considerarse usar el prasugrel por el mejor perfil de seguridad. Con el remdesivir no se ha registrado toxicidad cardiovascular mayor (29). En un reporte preliminar de una prueba aleatorizada controlada con placebo y doble ciego el uso de remdesivir fue superior para disminuir el tiempo a la recuperación (11 días vs. 15 para el grupo placebo), con una tendencia a menor mortalidad del grupo de tratamiento activo, pero algo que no fue estadísticamente significativo (32). El lopinavir ritonavir tampoco ha mostrado efectividad en pacientes con cuadros severos de COVID-19 (31)

\section{Trasplante cardiaco}

Se han reportado casos de COVID-19 en pacientes trasplantados, uno con compromiso leve y otro con un cuadro grave, cuyos síntomas fueron típicos. Estos fueron manejados con altas dosis de esteroides, inmunoglobulina intravenosa y antibióticos sin retirar la inmunosupresión de base, y no presentaron rechazo o muerte (33). Debe considerarse tamización a todos los donantes dado la prevalencia de infección asintomática por SARS-CoV 2. Durante la pandemia de H1N1 los potenciales donantes fueron tamizados en caso de tener síntomas o historia de exposición para así evitar la infección en el receptor o iniciar profilaxis en el donante si era positivo (34). Esta puede ser una estrategia razonable durante esta pandemia debido al número bajo de donantes de órganos en nuestro país.

\section{Conclusiones}

La pandemia actual ha puesto un reto a nuestra sociedad que va más allá de la capacidad del sistema sanitario. El personal de salud encargado del manejo de enfermedades cardiovasculares juega un papel primordial en la atención y tratamiento de la población afectada por la interacción encontrada entre estas enfermedades. En los meses futuros los estudios de investigación estarán dirigidos a mejorar nuestro entendimiento con respecto a los aspectos fisiopatológicos y terapéuticos. El eficiente uso de los recursos, del talento humano, la implementación de nuevas tecnologías y las medidas de salud pública serán aspectos invaluables que permitirán brindar una atención de mayor calidad, disminuyendo al máximo la propagación de la infección y los desenlaces fatales.

\section{Conflictos de interés}

Los autores manifiestan no tener conflictos de interés con el presente trabajo.

\section{Bibliografía}

1. Guan W-J, Ni Z-Y, Hu Y, Liang W-H, Ou C-Q, He J-X, et al. Clinical characteristics of Coronavirus Disease 2019 in China. N Engl J Med. 2020 30;382 (18):1708-20.

2. Huang C, Wang Y, Li X, Ren L, Zhao J, Hu Y, et al. Clinical features of patients infected with 2019 novel coronavirus in Wuhan, China. Lancet Lond Engl. 2020 15;395 (10223):497-506.

3. Wang D, Hu B, Hu C, Zhu F, Liu X, Zhang J, et al. Clinical Characteristics of 138 hospitalized patients with 2019 novel Coronavirus-infected pneumonia in Wuhan, China. JAMA. 2020 Mar 17;323 (11):1061-9. 
4. Epidemiology Working Group for NCIP Epidemic Response, Chinese Center for Disease Control and Prevention. The epidemiological characteristics of an outbreak of 2019 novel coronavirus diseases (COVID-19) in China. Zhonghua Liu Xing Bing Xue Za Zhi Zhonghua Liuxingbingxue Zazhi. 2020 Feb 10;41 (2):145-51.

5. Chen N, Zhou M, Dong X, Qu J, Gong F, Han Y, et al. Epidemiological and clinical characteristics of 99 cases of 2019 novel coronavirus pneumonia in Wuhan, China: a descriptive study. Lancet Lond Engl. 2020 15;395 (10223):507-13.

6. Liu K, Fang Y-Y, Deng Y, Liu W, Wang M-F, Ma J-P, et al. Clinical characteristics of novel coronavirus cases in tertiary hospitals in Hubei Province. Chin Med J (Engl). 2020 May 5;133 (9):1025-31.

7. Liu Y, Yang Y, Zhang C, Huang F, Wang F, Yuan J, et al. Clinical and biochemical indexes from 2019-nCoV infected patients linked to viral loads and lung injury. Sci China Life Sci. 2020;63 (3):364-74.

8. Zhang J-J, Dong X, Cao Y-Y, Yuan Y-D, Yang Y-B, Yan Y-Q, et al. Clinical characteristics of 140 patients infected with SARS-CoV-2 in Wuhan, China. Allergy. 2020;75 (7):1730-41.

9. Novel Coronavirus Pneumonia Emergency Response Epidemiology Team. [The epidemiological characteristics of an outbreak of 2019 novel coronavirus disea- ses (COVID-19) in China]. Zhonghua Liu Xing Bing Xue Za Zhi Zhonghua Liuxingbingxue Zazhi. 2020 Feb 17;41 (2):145-51.

10. Yang J, Zheng Y, Gou X, Pu K, Chen Z, Guo Q, et al. Prevalence of comorbidities and its effects in patients infected with SARS-CoV-2: a systematic review and meta-analysis. Int J Infect Dis IJID Off Publ Int Soc Infect Dis. 2020 May;94:91-5.

11. Yang $X, Y u Y, X u J$, Shu H, Xia J, Liu H, et al. Clinical course and outcomes of critically ill patients with SARS-CoV-2 pneumonia in Wuhan, China: a single-centered, retrospective, observational study. Lancet Respir Med. 2020;8 (5):475-81.

12. Zhou F, Yu T, Du R, Fan G, Liu Y, Liu Z, et al. Clinical course and risk factors for mortality of adult inpatients with COVID-19 in Wuhan, China: a retrospective cohort study. Lancet Lond Engl. 2020 28;395 (10229):1054-62.

13. Ruan Q, Yang K, Wang W, Jiang L, Song J. Clinical predictors of mortality due to COVID-19 based on an analysis of data of 150 patients from Wuhan, China. Intensive Care Med. 2020;46 (5):846-8.

14. Lippi G, Lavie CJ, Sanchis-Gomar F. Cardiac troponin I in patients with coronavirus disease 2019 (COVID-19): Evidence from a meta-analysis. Prog Cardiovasc Dis. 2020 Jun;63 (3):390-1.

15. Shi S, Qin M, Shen B, Cai Y, Liu T, Yang F, et al. Association of cardiac injury with mortality in hospitalized patients with COVID-19 in Wuhan, China. JAMA Cardiol. 2020 Jul 1;5 (7):802-10. 
16. Huang L, Zhao P, Tang D, Zhu T, Han R, Zhan C, et al. Cardiac involvement in patients recovered from COVID-2019 identified using magnetic resonance imaging. JACC Cardiovasc Imaging. 2020 May 12.

17. Puntmann VO, Carerj ML, Wieters I, Fahim M, Arendt C, Hoffmann J, et al. Outcomes of cardiovascular magnetic resonance imaging in patients recently recovered from Coronavirus Disease 2019 (COVID-19). JAMA Cardiol. 2020 Jul 27; doi: 10.1001/jamacardio.2020.3557. ahead of print.

18. Kwok CS, Gale CP, Kinnaird T, Curzen N, Ludman P, Kontopantelis E, et al. Impact of COVID-19 on percutaneous coronary intervention for ST-elevation myocardial infarction. Heart Br Card Soc. 2020 Aug 31; doi: 10.1136/heartjnl-2020-317650. Online ahead of print.

19. Garcia S, Stanberry L, Schmidt C, Sharkey S, Megaly M, Albaghdadi MS, et al. Impact of COVID-19 pandemic on STEMI care: An expanded analysis from the United States. Catheter Cardiovasc Interv Off J Soc Card Angiogr Interv. 2020 Aug 7; doi: 10.1002/ccd.29154. Online ahead of print.

20. Solomon MD, McNulty EJ, Rana JS, Leong TK, Lee C, Sung S-H, et al. The Covid-19 pandemic and the incidence of acute myocardial infarction. N Engl J Med. 2020 13:383 (7):691-3.

21. De Filippo O, D'Ascenzo F, Angelini F, Bocchino PP, Conrotto F, Saglietto A, et al. Reduced Rate of Hospital Admissions for ACS during Covid-19 Outbreak in Northern Italy. N Engl J Med. 2020 02;383 (1):88-9.

22. Shah K, Tang D, Ibrahim F, Ghosh B, Bhatti S, Akhabue E, et al. Surge in delayed myocardial infarction presentations: an inadvertent consequence of social distancing during the COVID-19 Pandemic. JACC Case Rep. 2020 Aug;2 (10):1642-7.

23. Choudry FA, Hamshere SM, Rathod KS, Akhtar MM, Archbold RA, Guttmann OP, et al. High thrombus burden in patients with COVID-19 presenting with ST-segment elevation myocardial infarction. J Am Coll Cardiol. 2020 08;76 (10):1168-76.

24. Bangalore S, Sharma A, Slotwiner A, Yatskar L, Harari R, Shah B, et al. ST-segment elevation in patients with Covid-19 - A Case Series. N Engl J Med. 2020 18;382 (25):2478-80.

25. Guo T, Fan Y, Chen M, Wu X, Zhang L, He T, et al. Cardiovascular Implications of fatal outcomes of patients with coronavirus disease 2019 (COVID-19). JAMA Cardiol. 2020 Jul 1;5 (7):811-8.

26. Arentz M, Yim E, Klaff L, Lokhandwala S, Riedo FX, Chong M, et al. Characteristics and outcomes of 21 critically ill patients with COVID-19 in Washington State. JAMA. 2020 28:323 (16):1612-4.

27. Vaduganathan M, Vardeny O, Michel T, McMurray JJV, Pfeffer MA, Solomon SD. Renin-angiotensin-aldosterone system inhibitors in patients with Covid-19. N Engl J Med. 2020 23;382 (17):1653-9. 
28. Yao X, Ye F, Zhang M, Cui C, Huang B, Niu P, et al. In Vitro Antiviral Activity and Projection of optimized dosing design of hydroxychloroquine for the treatment of severe acute respiratory syndrome coronavirus 2 (SARS-CoV-2). Clin Infect Dis Off Publ Infect Dis Soc Am. 2020 28;71 (15):732-9.

29. Driggin E, Madhavan MV, Bikdeli B, Chuich T, Laracy J, Biondi-Zoccai G, et al. Cardiovascular considerations for patients, health care workers, and health systems during the COVID-19 Pandemic. J Am Coll Cardiol. 2020 12;75 (18):2352-71.

30. Ray WA, Murray KT, Hall K, Arbogast PG, Stein CM. Azithromycin and the risk of cardiovascular death. N Engl J Med. 2012 May 17;366 (20):1881-90.

31. Chu CM, Cheng VCC, Hung IFN, Wong MML, Chan KH, Chan KS, et al. Role of lopinavir/ritonavir in the treatment of SARS: initial virological and clinical findings. Thorax. 2004 Mar;59 (3):252-6.

32. Beigel JH, Tomashek KM, Dodd LE, Mehta AK, Zingman BS, Kalil AC, et al. Remdesivir for the treatment of Covid-19 - Preliminary report. N Engl J Med. 2020 May 22; doi: 10.1056/NEJMoa2007764. Online ahead of print.

33. Li F, Cai J, Dong N. First cases of COVID-19 in heart transplantation from China. J Heart Lung Transplant Off Publ Int Soc Heart Transplant. 2020;39 (5):496-7.

34. Danziger-Isakov LA, Husain S, Mooney ML, Hannan MM, ISHLT Infectious Diseases Council. The novel 2009 H1N1 influenza virus pandemic: unique considerations for programs in cardiothoracic transplantation. J Heart Lung Transplant Off Publ Int Soc Heart Transplant. 2009 Dec;28 (12):1341-7. 